\title{
Evaluation of the Frequency and Pathological Diagnosis of Vulvar Lesions Is it Associated with Vulvar Pruritus - If So, How Closely?
}

\author{
Omer DEMIR¹, Mirac OZALP1, Turhan ARAN', Mehmet Armagan OSMANAGAOGLU1
}

Trabzon, Turkey

\section{ABSTRACT}

OBJECTIVE: Vulvar lesions are not well known to gynecologists and often cause difficulties in the differential diagnosis. In this study; we aimed to show the frequency of pathologic diagnosis of vulvar lesions and their relationship with vulvar pruritus.

STUDY DESIGN: The pathological results of the patients who underwent vulvar biopsy between 2015 and 2019 were examined. The frequency of vulvar lesions was determined and classified under certain headings for gynecologists. The pathological diagnoses were correlated with the severity of pruritus.

RESULTS: A total of 220 patients underwent a vulvar biopsy between the dates indicated in the study. The mean age of these patients was $47.9 \pm 16.7$. The most common concomitant lesion was vulvar pruritus with a rate of $62.7 \%$ in cases who were referred to the outpatient clinic and referred for vulvar biopsy. According to biopsy results, the three most common pathological diagnoses were proliferative lesions, infection, and dermatoses. Dermatoses were the most common pathologic diagnosis in patients with severe pruritus.

CONCLUSION: Thanks to the clear classification of vulvar lesions for gynecologists; pathological diagnoses will not be overlooked and differential diagnoses will be more effective. In vulvar lesions accompanied by pruritus, making the differential diagnosis according to the severity of pruritus and bringing the possible diagnosis to mind first will speed up the treatment.

Keywords: Biopsy, Classification, Pruritus, Vulva

Gynecol Obstet Reprod Med 2021;27(3):273-278

\section{Introduction}

A wide spectrum of benign, premalignant, and malignant lesions may occur on the vulva. However, it may sometimes be difficult for the clinician to recognize certain lesions with which he/she is not familiar or of which he/she is unable to identify the dermatological features. The way to overcome this is to acquire a mastery of vulvar anatomy, to know the fre-

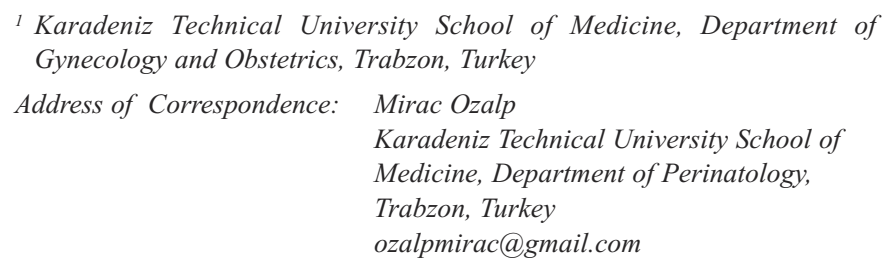

Submitted for Publication: 16.09.2020 Revised for Publication: 19.09.2020 Accepted for Publication: 27.10.2020 Online Published: 07.01.2021 ORCID IDs of the authors: $\quad$ OD: 0000-0003-4376-8733 MO: 0000-0002-2255-1642, TA: 0000-0001-9488-9776 MAO: 0000-0001-5115-5262

\begin{tabular}{c|c}
\hline Quick Response Code: & Access this article online \\
\cline { 2 - 2 } $\begin{array}{c}\text { Website: www.gorm.com.tr } \\
\text { e- mail: info@gorm.com.tr }\end{array}$ \\
\cline { 2 - 2 }
\end{tabular}

How to cite this article: Demir O. Ozalp M. Aran T. Osmanagaoglu MA. Evaluation of the Frequency and Pathological Diagnosis of Vulvar Lesions is it Associated with Vulvar Pruritus-If So, How Closely? Gynecol Obstet Reprod Med 2021;27(3):273-278 quency of lesions that can be seen in the vulva, and to know which features should be considered in the distinction between benign and malignant.

For the patient presenting with any symptoms, the first stage of the evaluation is to take a comprehensive medical history. Ideally, history should be taken before the removal of the patient's clothing for a physical evaluation. In addition, as opposed to a routine medical history, taking a site-specific anamnesis enables diagnosis and elucidation of the etiology (1). Vulvar symptoms that lead the clinician may include a palpable mass, discoloration, burning, and itching. The most common of these is vulvar pruritus and has been associated with many lesions (1).

The vulva is the junction of tissues originating from the three embryological layers (endoderm, mesoderm, and ectoderm). The tissues originating from these three germ layers are different in structure as well as hormonal sensitivities, immune responses, and neural distribution (2). Therefore, the location of the lesion is important in terms of diagnosis as well as treatment.

The vulva is a region open to many foreign proteins and allergens. Therefore, immune responses may vary (2). 
If there is a lesion in the vulva, or if the patient presents with any complaint, the location, type, and relationship of this lesion with other systemic and laboratory findings are important for diagnosis and treatment.

This study aims to investigate the frequency of vulvar lesions, to examine the relationship between pruritus and the severity of pruritus, to increase awareness of vulvar lesions and to emphasize the importance of biopsy.

\section{Material and Method}

The study was designed as a retrospective analysis of the data of patients at the Karadeniz Technical University Faculty of Medicine, Department of Obstetrics and Gynecology who underwent vulvar biopsy between 2015-2019.

All patients who present to the gynecology outpatient clinic for any reason undergo a detailed history and routine vulvovaginal examination. After this combined evaluation, a vulvar biopsy is performed to obtain a definitive diagnosis of patients who have vulvar symptoms such as a palpable vulvar lesion, discoloration, vulvar pruritus, and pathology observed during vulvovaginal examinations.

In this study, the data of all patients who underwent a vulvar biopsy were reviewed retrospectively and the relationship between the symptoms and diagnosis and the frequency of the pathological diagnoses were investigated. Vulvar biopsies were obtained from patients with vulvar palpable lesions, treatment-resistant vulvar pruritus, and abnormal color changes in the vulva.

In addition, patients were questioned about the presence and severity of pruritus. The severity of pruritus was divided into three grades; mild, moderate, and severe. The mild form was accepted as occasional pruritus experienced during the day which was not very severe. The moderate form is defined as persistent and uncomfortable pruritus. The severe form is characterized by itching that rouses one from sleep at night. The results of the cases with pruritus and vulvar biopsies were grouped according to the severity of pruritus and an attempt was made to establish a differential diagnosis of the vulvar lesions according to the severity of pruritus.

The patients whose presence or severity of pruritis could not be reached in retrospective screening were called and the presence and severity of symptoms were questioned.

The study was conducted with the approval of the Faculty Ethics Committee (\#287/2019) and necessary permissions were obtained from the head physician for use of the patient data. The study was created based on the principles set out in the Declaration of Helsinki.

\section{Statistical analysis}

The Statistical Package for the Social Sciences (SPSS) version 21 software program designed for Windows was used for statistical analysis. All continuous variables were defined as mean and standard deviations. Categorical variables were shown as a percentage of the total group.

\section{Results}

A total of 220 patients underwent a vulvar biopsy between the dates indicated in the study. The mean age of these patients was $47.9 \pm 16.7$. The number of postmenopausal patients was 98 and constituted $44.5 \%$ of the total number. The use of vulvar cosmetic products was identified in $7.7 \%$ of patients (one patient with genital melanosis, two patients with Bartholin cyst excision, and five patients with chronic inflammation had a history of cosmetic use). Fourteen patients (6.4\%) were diagnosed with type 2 diabetes. The most common concomitant symptom was vulvar pruritus with a rate of $62.7 \%$ in cases who were referred to the outpatient clinic and referred for vulvar biopsy. The demographic characteristics of the 220 patients are shown in table I.

Table I: The demographic characteristics of the 220 patients

\begin{tabular}{ll}
\hline Age (years-mean+/-sd) (min-max) & $47.9 \pm 16.7$ (20-91) \\
Menopausal status & \\
$\quad$ Premenopausal-n/\% & $122 / 55.5 \%$ \\
$\quad$ Postmenopausal-n/\% & $98 / 44.5 \%$ \\
Using vulvar cosmetic products-n/\% & $17 / 7.7 \%$ \\
Diagnosed with Diabetes mellitus & $14 / 6.4 \%$ \\
Using antibiotics before diagnosis & $14 / 6.4 \%$ \\
\hline
\end{tabular}

The vulvar biopsy results were characterized under 10 main headings. These were; vulvar dermatoses, epidermal/ dermal solid vulvar lesions, vulvar lesions caused by an infection agent, cystic vulvar lesions, vulvar malignancies, vulvar proliferative lesions, vulvar ulcerative lesions, vulvar melanocytic lesions, vulvar vascular lesions, and chronic inflammation not included in other groups resulting in vulvar lesions. Each of these is divided into subgroups. These main groups and subgroups, the number and percentage of patients in these groups, and their relationship with vulvar pruritus are as shown in table II.

When the vulvar biopsy pathology results were examined, the first three found according to frequency were: vulvar proliferative lesions, vulvar lesions caused by infectious agents, and vulvar dermatoses $(29.1 \%, 28.2 \%$, and $12.7 \%$, respectively). In these main groups, it was seen that the most common association with pruritus was vulvar lesions caused by infection agents with $38.4 \%$, and the most common subtype was vulvar candidiasis (26.8\%).

When the symptom severity of patients with concomitant pruritus was questioned; 73 patients $(52.9 \%)$ had mild pruritus, 42 patients $(30.4 \%)$ had moderate pruritus and 23 patients $(16.7 \%)$ had severe pruritus which woke them from sleep at night, and the number and percentage of these patients are shown in table III. 
Table II: Vulvar biopsy results and its relationship with pruritus

\begin{tabular}{|c|c|c|c|c|c|c|c|}
\hline & & & $\mathrm{N}$ & $\%$ & Pruritus+ & $\% *$ & $\%$ ** \\
\hline \multirow[t]{5}{*}{ Vulvar Proliferative Lesions } & & & 64 & 29.1 & 29 & 21 & 45.3 \\
\hline & & Inflammatory granulation tissue & 17 & 7.7 & 17 & 12.3 & 100 \\
\hline & & Squamous hyperplasia & 16 & 7.7 & - & & \\
\hline & & Squamous papilloma & 16 & 7.3 & - & & \\
\hline & & Fibroepithelial polyp & 15 & 6.8 & 12 & 8.7 & 80 \\
\hline \multirow{5}{*}{\multicolumn{2}{|c|}{$\begin{array}{l}\text { Vulvar lesions caused by an } \\
\text { infectious agent }\end{array}$}} & & 62 & 28.2 & 53 & 38.4 & 85.5 \\
\hline & & Vulvar candidiasis & 37 & 16.8 & 37 & 26.8 & 100 \\
\hline & & Vulvar condyloma acuminata & 21 & 9.5 & 14 & 10.1 & 66.7 \\
\hline & & Molluscum contagiosum & 3 & 1.4 & 1 & 0.7 & 33.3 \\
\hline & & Furunculosis & 1 & 0.5 & 1 & 0.7 & 100 \\
\hline \multirow[t]{12}{*}{ Vulvar Dermatoses } & & & 28 & 12.7 & 28 & 20.3 & 100 \\
\hline & Spongiotic pattern & & 11 & 5 & 11 & 8 & 100 \\
\hline & & Irritant contact dermatitis & 6 & 2.7 & 6 & 4.3 & 100 \\
\hline & & Allergic contact dermatitis & 3 & 1.4 & 3 & 22 & 100 \\
\hline & & Perivascular dermatitis & 2 & 0.9 & 2 & 1.4 & 100 \\
\hline & Lichenoid pattern & & 10 & 4.5 & 10 & 7.2 & 100 \\
\hline & & Lichen sclerosis et atrophicus & 9 & 4.0 & 9 & 6.5 & 100 \\
\hline & & Lichen planus - erosive type & 1 & 0,5 & 1 & 0.7 & 100 \\
\hline & Acantotic pattern & & 6 & 2.7 & 6 & 4.3 & 100 \\
\hline & & Lichen simplex chronicus & 6 & 2.7 & 6 & 4.3 & 100 \\
\hline & Granulomatous pattern & & 1 & 0.5 & 1 & 0.7 & 100 \\
\hline & & $\begin{array}{l}\text { Foreign body type giant } \\
\text { cell reaction }\end{array}$ & 1 & 0.5 & 1 & 0.7 & 100 \\
\hline \multirow[t]{3}{*}{ Cystic vulvar lesion } & & & 27 & 12.3 & 3 & 2.2 & 11.1 \\
\hline & & Bartholin cyst & 20 & 9.1 & 3 & 2.2 & 15 \\
\hline & & Epidermoid cyst & 7 & 3.2 & - & & \\
\hline \multirow[t]{4}{*}{ Vulvar malignancy } & & & 12 & 5.5 & 8 & 5.8 & 66.6 \\
\hline & & Squamous cell carcinoma & 10 & 4.5 & 8 & 5.8 & 80 \\
\hline & & Malignant melanoma & 1 & 0.5 & - & & \\
\hline & & Malignant mesenchymal tumor & 1 & 0.5 & - & & \\
\hline \multirow[t]{4}{*}{ Epidermal/dermal solid vulvar lesion } & & & 7 & 3.2 & 5 & 3.6 & 71.4 \\
\hline & & Seborrheic keratosis & 3 & 1.4 & 3 & 2.2 & 100 \\
\hline & & Syringoma & 2 & 0.9 & 2 & 1.4 & 100 \\
\hline & & Lipoma & 2 & 0.9 & - & & \\
\hline \multirow[t]{4}{*}{ Vulvar melanocytic lesion } & & & 7 & 3.2 & 2 & 1.4 & 28.6 \\
\hline & & Intradermal nevus & 3 & 1.4 & - & & \\
\hline & & Compound nevus & 2 & 0.9 & - & & \\
\hline & & Vulvar melanosis & 2 & 0.9 & 2 & 1.4 & 100 \\
\hline \multirow[t]{2}{*}{ Vulvar vascular lesion } & & & 3 & 1.4 & - & & \\
\hline & & Cavernous hemangioma & 3 & 1.4 & - & & \\
\hline \multirow[t]{2}{*}{ Vulvar ulcerative lesion } & & & 2 & 0.9 & 2 & 1.4 & 100 \\
\hline & & Genital ulcer & 2 & 0.9 & 2 & 1.4 & 100 \\
\hline \multirow[t]{2}{*}{ Unclassified vulvar inflammation } & & & 8 & 3.6 & 8 & 5.8 & 100 \\
\hline & & Chronic inflammation & 8 & 3.6 & 8 & 5.8 & 100 \\
\hline Total & & & 220 & 100 & 62.7 & & \\
\hline
\end{tabular}

${ }^{*}$ Percentage of the patient who described pruritus in the specified diagnosis in the total patient who described pruritus

${ }^{* *}$ Percentage of the number of patients describing pruritus in the specified diagnosis in the total patient with the same diagnosis 
Table III: Distribution of diagnoses and percentages in patients according to the severity of pruritus

\begin{tabular}{lll}
\hline $\begin{array}{l}\text { Group with severe pruritus } \\
\mathbf{n}=\mathbf{2 3}(\mathbf{1 6 . 7 \% )}\end{array}$ & $\mathbf{n / N}$ & $\%$ \\
\hline Lichen sclerosis et atrophicus & $9 / 9$ & 100 \\
Lichen simplex chronicus & $6 / 6$ & 100 \\
Genital ulcer & $2 / 2$ & 100 \\
Lichen planus- erosive type & $1 / 1$ & 100 \\
Irritant contact dermatitis & $2 / 6$ & 33.3 \\
Vulvar candidiasis & $3 / 37$ & 8.1
\end{tabular}

Group with moderate pruritus $n=42(30.4 \%)$

$\begin{array}{lll}\text { Allergic contact dermatitis } & 3 / 3 & 100 \\ \text { Foreign body type giant cell reaction } & 1 / 1 & 100 \\ \text { Furunculosis } & 1 / 1 & 100 \\ \text { Vulvar candidiasis } & 29 / 37 & 78.4 \\ \text { Irritant contact dermatitis } & 4 / 6 & 66.7 \\ \text { Bartholin cyst } & 1 / 20 & 5 \\ \text { Vulvar condyloma acuminata } & 1 / 21 & 4.8\end{array}$

\section{Group with mild pruritus $n=73(52.9 \%)$}

Inflammatory granulation tissue

Seborrheic keratosis

Perivascular dermatitis

Vulvar melanosis

Syringoma

Fibroepithelial polyp

Squamous cell carcinoma

Chronic inflammation

Vulvar condyloma acuminata

Molluscum contagiosum

Vulvar candidiasis

Bartholin cyst

$\begin{array}{ll}17 / 17 & 100 \\ 3 / 3 & 100 \\ 2 / 2 & 100 \\ 2 / 2 & 100 \\ 2 / 2 & 100 \\ 12 / 15 & 80 \\ 8 / 10 & 80 \\ 6 / 8 & 75 \\ 13 / 21 & 61.9 \\ 1 / 3 & 33.3 \\ 5 / 37 & 13.5 \\ 2 / 20 & 10\end{array}$

$n$ : The number of patients with pruritus at the specified diagnosis

$N$ : The number of patients at the specified diagnosis

Table III also shows the distribution of the biopsy results of the patients who described mild, moderate, and severe pruritus respectively, and the percentage of pruritus within these results.

Almost all subgroups of the vulvar dermatosis group presented to the outpatient clinic due to severe pruritus, $78.4 \%$ of patients diagnosed with vulvar candidiasis described moderate pruritus, $61.9 \%$ of patients with vulvar condyloma presented with mild pruritus and severe pruritus was rare in this group.

Mild pruritus was seen in $80 \%$ of patients with squamous cell carcinoma. However, no vulvar malignancy accompanied by moderate or severe pruritus was observed.

In addition, two patients with vulvar ulcers had severe pruritus.

\section{Discussion}

There are certain differences between dermatological and gynecological approaches to vulvar lesions. Dermatologists classify vulvar lesions according to the International Vulvovaginal Diseases Working Group (ISSVD) classification and tend to communicate with each other more readily (3). However, gynecologists do not categorize themselves as dermatologists when evaluating vulvar lesions. For this reason, gynecologists who do not have a universal approach towards the evaluation of vulvar lesions tend to stay a little distant from these diseases and generally toss the ball to dermatologists. Since the approach of gynecologists to these diseases does not go beyond classifying them as benign, premalignant, and malignant lesions, it can be said that they are not very familiar with dermatological and pathological diagnoses.

Since the study data is from a gynecology clinic which primarily evaluates vulvar lesions in the faculty, the aim was to determine the place of vulvar lesions in gynecology practice, to see how the indications for biopsies are placed and the distribution of these indications and to show the relationship between these lesions and pruritus which is the most common concomitant symptom. It is thought that the results will be applicable in gynecology practice. The two most important considerations in the diagnosis of vulvar lesions are; the medical history and careful genital examination. If this pair is supported by a vulvar biopsy, then the diagnosis becomes definite and treatment can be provided accordingly.

Asymmetry, border irregularity, discoloration, rapid change, hemorrhage, and non-healing ulcers raise the suspicion of malignancy, so a vulvar biopsy is recommended $(4,5)$. In addition, a vulvar biopsy is recommended in patients with lichen sclerosis who have developed a new lesion after the standard treatment given at the initial evaluation has failed to work (6). Since vulva biopsy is a procedure performed under local anesthesia in polyclinic conditions, it should not be avoided and should be consulted in cases where there is the slightest suspicion of malignancy. In the current study, a vulvar biopsy was taken from 220 patients and vulvar malignancy was detected in 12 patients (5.5\%). Early diagnosis by biopsy is of great importance where there is a suspicion of vulvar cancer which rates as $5 \%$ of all gynecological malignancies $(7,8)$.

In the current study, vulvar lesions were classified in a way that could be understood by all clinicians. Due to the difficulty in understanding gynecologists' dermatological classifications, vulvar lesions were classified under 10 subheadings. According to the biopsy results, the largest group was $29.1 \%$ : Inflammatory granulation tissue, squamous hyperplasia, squamous papilloma, and fibroepithelial polyps constitute the vulvar proliferative lesion group. The second group is the vulvar lesion formed by infectious agents with a rate of $28.2 \%$ (the most common subgroup is candidiasis). The third most common group was vulvar dermatoses (12.7\%).

This group is of great importance to gynecologists. As there are premalignant lesions in this group that can regress when treated, and if they remain untreated, they may progress to ma- 
lignancy (9). As can be seen from the results of the study, almost all of this group presented with pruritus. In the evaluations performed, vulvar examinations revealed excoriated and lichenified areas. Due to their history of severe itching and subject to a physical examination, a biopsy should be taken from patients with suspected dermatosis and treatment should be started immediately if the diagnosis is confirmed. Lichen sclerosis is one of the risk factors for vulvar malignancy and it has been shown in the literature that vulvar cancer develops in a background of lichen sclerosis at a rate of $2-5 \%(10,11)$. In the current study, lichen sclerosis was found in $4 \%$ of the 220 patients, all of whom were postmenopausal and all accompanied by severe pruritus. After proper treatment and regular controls, vulvar malignancy can be prevented.

In addition, the lesions were classified according to the severity of vulvar pruritus and the aim to make the differential diagnosis easier based on the severity of pruritus at the time of admission to the clinic. Accordingly, in patients with severe itching, vulvar dermatoses (especially lichen sclerosis) and genital ulcers should be considered. A biopsy revealed two genital ulcers. These patients were diagnosed with Behçet's disease after joint evaluations with the dermatology and rheumatology units and their treatment was begun. In other words, an immunological disease was diagnosed from the vulvar lesion (12). The importance of evaluation of vulvar lesions can be better understood having regard to these two cases.

In the current study, it was found that the pathological problem in women who described moderate itching was mostly vulvar candidiasis and allergic and irritant contact dermatitis. The most striking group in the group of patients who described mild itching was the malignancy group. In $80 \%$ of the women with SCC have mild pruritus, the remaining $20 \%$ did not experience itching.

Fourteen patients (6.4\%) who had undergone a vulvar biopsy had a history of taking antibiotics for other reasons before admission and all of these patients were reported as having vulvar candidiasis. This shows that the use of systemic antibiotics causes the proliferation of pathogenic microorganisms due to the change in genital flora, which is a good example of how anamnesis can be associated with pathological diagnosis (13).

When the glycemic controls of candidiasis patients were checked, it was found that $6.4 \%$ had uncontrolled diabetes. In this way, patients were diagnosed with diabetes from vulvar lesions.

Poor glycemic control is one of the most serious risk factors that predispose a woman to genital fungal diseases, which was evident in this study $(14,15)$.

When the patients were questioned as to whether they used extra cosmetic products for vulvar hygiene while taking their anamnesis, it was seen that $7.7 \%$ of them were using such products. All the patients whose pathological evaluation resulted from irritant or allergic contact dermatitis provided this same history. In addition, one patient with genital melanosis, two patients with Bartholin cyst excision, and five patients with chronic inflammation had a history of cosmetic use. In the literature, it is stated that there is a history of the use of cosmetics in infectious situations leading to chronic inflammation $(16,17)$.

The strength of our research is the classification of vulvar lesions according to the severity of pruritus, and this classification provides the opportunity for gynecologists to make an easy differential diagnosis. The limitations of the study are its retrospective nature and the limited number of patients.

In conclusion, if the gynecologists' awareness of vulvar lesions is increased and this subject is not considered as only the work of dermatologists, new approaches can be developed and early prevention of malignancy can be achieved. Even during routine gynecological examinations, the vulvar region should be carefully examined, and the mere statement of symptoms should not be accepted as definitive. In addition, a gynecologist who understands the different pathologies that can be diagnosed in the presence of vulvar pruritus, the most commonly expressed symptom, can help the patient more and provide more effective symptom control with early diagnosis. Thanks to this classification we have made, vulvar lesion classification can be made easier with pruritus severity and gynecologists will approach vulvar lesions more confidently.

\section{Acknowledgment: None}

Funding statement: No funding was received for this study. Conflicts of Interest: The authors declare that they have no conflicts of interest.

Author contribution: OD: Project development, data collection, analysis, interpretation of data, manuscript writing, revising the manuscript. MO: Analysis, interpretation of data, manuscript writing, revising the manuscript. TA: Project manager, critical analysis. MAO: Project manager

\section{References}

1. ACOG Practice Bulletin No. 93: diagnosis and management of vulvar skin disorders. Obstet Gynecol. 2008; 111(5):1243-53. Reaffirmed 2019. Doi:10.1097/AOG. 0b013e31817578ba.

2. Yeung J, Pauls RN. Anatomy of the vulva and the female sexual response. Obstet Gynecol Clin North Am. 2016;43(1):27-44. Doi:10.1016/j.ogc.2015.10.011.

3. Lynch PJ, Moyal-Barracco M, Bogliatto F, Micheletti L, Scurry J. 2006 ISSVD classification of vulvar dermatoses: pathologic subsets and their clinical correlates. J Reprod Med. 2007;52(1):3-9. PMID:17286059.

4. Sand FL, Thomsen SF. Skin diseases of the vulva: eczematous diseases and contact urticaria. J Obstet Gynaecol. 2018;38(3):295-300. Doi:10.1080/01443615. 
2017.1329283.

5. Sand FL, Thomsen SF. Skin diseases of the vulva: Infectious diseases. J Obstet Gynaecol. 2017;37(7):840-8. Doi:10.1080/01443615.2017.1306696.

6. Hoang MP, Reutter J, Papalas JA, Edwards L, Selim MA. Vulvar inflammatory dermatoses: an update and review. Am J Dermatopathol. 2014;36(9):689-704. Doi:10.1097/ DAD.0000000000000149.

7. Siegel RL, Miller KD, Jemal A. Cancer Statistics, 2017. CA Cancer J Clin. 2017;67(1):7-30. Doi:10.3322/caac. 21387.

8. Schuurman MS, van den Einden LC, Massuger LF, Kiemeney LA, van der Aa MA, de Hullu JA. Trends in incidence and survival of Dutch women with vulvar squamous cell carcinoma. Eur J Cancer. 2013;49(18):3872-80. Doi: 10.1016/j.ejca.2013.08.003.

9. Yang EJ, Kong CS, Longacre TA. Vulvar and anal intraepithelial neoplasia: Terminology, diagnosis, and ancillary studies. Adv Anat Pathol. 2017;24(3):136-50. Doi: 10.1097/PAP.0000000000000149.

10. Bleeker MC, Visser PJ, Overbeek LI, van Beurden M, Berkhof J. Lichen Sclerosus: Incidence and risk of vulvar squamous cell carcinoma. Cancer Epidemiol Biomarkers Prev. 2016;25(8):1224-30. Doi:10.1158/1055-9965.EPI-
16-0019.

11. Thomas RH, Ridley CM, McGibbon DH, Black MM. Anogenital lichen sclerosus in women. J R Soc Med. 1996;89(12):694-8. PMID:9014881.

12. Lewis FM, Velangi SS. An overview of vulvar ulceration. Clin Obstet Gynecol. 2005;48(4):824-37. Doi:10.1097/ 01.grf.0000179669.21811.8f.

13. Wilton L, Kollarova M, Heeley E, Shakir S. Relative risk of vaginal candidiasis after use of antibiotics compared with antidepressants in women: postmarketing surveillance data in England. Drug Saf. 2003;26(8):589-97. Doi: 10.2165/00002018-200326080-00005.

14. Donders GG. Lower genital tract infections in diabetic women. Curr Infect Dis Rep. 2002;4(6):536-9. Doi:10. 1007/s11908-002-0042-y.

15. de Leon EM, Jacober SJ, Sobel JD, Foxman B. Prevalence and risk factors for vaginal Candida colonization in women with type 1 and type 2 diabetes. BMC Infect Dis. 2002;2:1. Doi:10.1186/1471-2334-2-1.

16. Foxman B. The epidemiology of vulvovaginal candidiasis: risk factors. Am J Public Health. 1990;80(3):329-31. Doi:10.2105/ajph.80.3.329.

17. Sobel JD. Vulvovaginal candidosis. Lancet. 2007;369 (9577):1961-71. Doi:10.1016/S0140-6736(07)60917-9. 